\title{
Density-matrix theory for the ground state of spin-compensated harmonically confined two-electron model atoms with general interparticle repulsion
}

\author{
Ali Akbari, ${ }^{1}$ Norman H. March, ${ }^{1,3,4}$ and Angel Rubio ${ }^{1,2}$ \\ ${ }^{1}$ Nano-Bio Spectroscopy Group and ETSF Scientific Development Centre, Departamento de Física de Materiales, \\ Universidad del País Vasco, Centro de Física de Materiales CSIC-UPV/EHU-MPC and DIPC, Av. Tolosa 72 , \\ E-20018 San Sebastián, Spain \\ ${ }^{2}$ Fritz-Haber-Institut der Max-Planck-Gesellschaft, Berlin, Germany \\ ${ }^{3}$ Department of Physics, University of Antwerp (RUCA), Groenenborgerlaan 171, B-2020,, Antwerp, Belgium \\ ${ }^{4}$ Oxford University, Oxford, England
}

(Received 5 December 2008; published 11 September 2009)

\begin{abstract}
For model two-electron atoms with harmonic confinement, the correlated first-order density matrix can be expressed in terms of the relative motion wave function $\Psi_{R}(r)$. Here we demonstrate that the probability density $P(r)$ associated with this wave function is directly related to the x-ray scattering factor $f(G)$. This latter quantity, in turn, is determined by the ground-state electron density $n(r)$. The Euler-Lagrange equation of the resulting density-matrix theory is thereby shown to take the form of a third-order integro-differential equation for $n(r)$ in which the probability density $P(r)=\Psi_{R}^{2}(r)$ also appears. For two specific choices of the interaction between the two fermions under consideration, the above integro-differential equation derived here is shown to lead back to known linear homogeneous differential equations for the electron density. Finally, it is emphasized that specific equations summarized here will apply directly to theoretical study of the nonrelativistic groundstate electron density $n(r, Z)$ in the He-like ions with atomic number $Z$.
\end{abstract}

DOI: 10.1103/PhysRevA.80.032509

PACS number(s): 31.15.ve

\section{INTRODUCTION AND BACKGROUND}

The study of variational methods based on the first-order density matrix goes back at least to the work of March and Young $[1,2]$. These authors were concerned with one-body problems characterized by the idempotent Dirac density matrices [3], while in [1] $N$-fermion systems were studied, within such an independent electron framework. There is now considerable interest in density-matrix theory for interacting electrons and the present study falls within this area. However, we here treat the family of two-electron model atoms referred to in the title, as proposed by Holas et al. [4]. While our main focus below is the correlated first-order density matrix $\gamma\left(\mathbf{r}_{1}, \mathbf{r}_{2}\right)$, let us begin with its diagonal form [4]

$$
\begin{aligned}
n(r)= & \left.\gamma\left(\mathbf{r}_{1}, \mathbf{r}_{2}\right)\right|_{\mathbf{r}=\mathbf{r}_{1}=\mathbf{r}_{2}} \\
= & \frac{8}{\pi^{1 / 2}} \exp \left(-\frac{r^{2}}{a^{2}}\right) \int_{0}^{\infty} d y y^{2} \exp \left(-\frac{y^{2}}{4}\right) \\
& \times\left[\Psi_{R}(a y)\right]^{2} \frac{\sinh \left(\frac{r y}{a}\right)}{\left(\frac{r y}{a}\right)} .
\end{aligned}
$$

Here the length $a=(\hbar / 2 m \omega)^{1 / 2}$, where the harmonic confinement potential is $\frac{1}{2} m \omega^{2} r^{2}$, with $m$ as the electron mass. The total ground-state wave function has the spatial form

$$
\Psi\left(\mathbf{r}_{1}, \mathbf{r}_{2}\right)=\Psi_{C}\left(\frac{\left|\mathbf{r}_{1}+\mathbf{r}_{2}\right|}{2}\right) \Psi_{R}\left(\left|\mathbf{r}_{1}-\mathbf{r}_{2}\right|\right)
$$

where $C$ denotes center of mass and $R$ denoted the relative motion. It will be useful below to re-express $R$ and $C$ coordinates appearing in Eq. (2) by

$$
\mathbf{b}=\mathbf{r}_{1}-\mathbf{r}_{2}, \quad \mathbf{c}=\left(\mathbf{r}_{1}+\mathbf{r}_{2}\right) / 2 .
$$

We next construct the so-called atomic scattering factor $f(G)$ defined as the Fourier transform of the ground-state density $n(r)$,

$$
f(G)=\int n(r) \exp (i \mathbf{G} \cdot \mathbf{r}) d \mathbf{r} .
$$

Then one readily obtains by employing Eqs. (1) and (4) the result that

$$
f(G)=\frac{16 \pi}{G} \exp \left(-\frac{G^{2} a^{2}}{4}\right) \int_{0}^{\infty} y \sin \left(\frac{G y}{2}\right)\left[\Psi_{R}(y)\right]^{2} d y .
$$

We note next that the relative motion wave function $\Psi_{R}$ appearing in Eq. (5) satisfies the Schrödinger-like equation [4],

$$
\left[-\frac{\hbar^{2}}{m} \nabla^{2}+V_{e f f}(r)\right] \Psi_{R}(r)=E_{R} \Psi_{R}(r),
$$

where the effective potential energy $V_{e f f}(r)$ is given by

$$
V_{e f f}(r)=\frac{1}{4} m \omega^{2} r^{2}+u(r)
$$

while $E_{R}$ is the energy of the relative motion. The above equations all hold for a general interparticle interaction $u(r)$ in the harmonically confined family of two-electron model atoms. We now turn to consider the off-diagonal extension of the above result (1). For general density-matrix theory then we have from relation (17) in [4] that the correlated firstorder density matrix $\gamma\left(\mathbf{r}_{1}, \mathbf{r}_{2}\right)$, using the vectors $\mathbf{b}$ and $\mathbf{c}$ defined in Eq. (3), is given by 


$$
\begin{aligned}
\gamma\left(\mathbf{r}_{1}, \mathbf{r}_{2}\right)= & \gamma(\mathbf{b}, \mathbf{c}) \\
= & 2 \int d \mathbf{x} \Psi_{C}\left(\frac{1}{2}\left|\mathbf{x}+2 \mathbf{c}+\frac{1}{2} \mathbf{b}\right|\right) \\
& \times \Psi_{C}\left(\frac{1}{2}\left|\mathbf{x}+2 \mathbf{c}-\frac{1}{2} \mathbf{b}\right|\right) \Psi_{R}\left(\left|\mathbf{x}+\frac{1}{2} \mathbf{b}\right|\right) \\
& \times \Psi_{R}\left(\left|\mathbf{x}-\frac{1}{2} \mathbf{b}\right|\right) .
\end{aligned}
$$

Here the center-of-mass wave function $\Psi_{C}(r)$ is known once for all for this family of model atoms as

$$
\Psi_{C}(r)=\frac{1}{a^{3 / 2} \pi^{3 / 4}} \exp \left(-\frac{r^{2}}{2 a^{2}}\right),
$$

where the length $a$ is given following Eq. (1). With this background, we proceed below to develop an exact densitymatrix theory based on Eqs. (1) and (8) for the family of models summarized in the title. From Eq. (5), $\Psi_{R}^{2}(r)$ entering Eq. (1) is known in terms of $f(G)$.

\section{EULER-LAGRANGE EQUATION OF DENSITY- MATRIX THEORY AS AN INTEGRO-DIFFERENTIAL EQUATION FOR GROUND-STATE FERMION DENSITY $n(r)$}

To introduce the derivation of the Euler-Lagrange equation of the density matrix for this family, let us write the known expression for the difference in kinetic-energy density $t_{L}(r)-t_{g}(r)$ in terms of the scattering factor $f(G)$. Here $t_{L}$ denotes the Laplacian form $\psi \nabla^{2} \psi$ in terms of wave functions, while $t_{g}$ is the gradient alternative $(\nabla \psi)^{2}$. The difference $t_{g}-t_{L}$ is given by

$$
t_{L}(r)-t_{g}(r)=-\frac{\hbar^{2}}{4 m} \nabla^{2} n(r) .
$$

Then it follows from Eq. (4) that

$$
t_{L}(r)-t_{g}(r)=\frac{\hbar^{2}}{4 m(2 \pi)^{3}} \int G^{2} f(G) e^{i \mathbf{G} \cdot \mathbf{r}} d \mathbf{G} .
$$

Below in Sec. III, we use a version of this Eq. (11) to obtain specific results for special cases of the fermion-fermion (ff) interaction $u\left(r_{12}\right)$ in the present class of harmonically confined particles. Equation (11) of course can be inverted to read as

$$
G^{2} f(G)=\frac{4 m}{\hbar^{2}} \int\left[t_{L}(r)-t_{g}(r)\right] e^{-i \mathbf{G} \cdot \mathbf{r}} d \mathbf{r} .
$$

This suggests that when $f(G)$ is available (see examples in [5]), an elegant route to say $t_{g}(r)$ [6] should be via these two equations above.

However, and quite generally, by differentiation of the definition of the density $n(r)$ from the total wave function $\Psi\left(\mathbf{r}, \mathbf{r}_{2}\right)$, March et al. [7] obtained straightforwardly the identity

$$
\begin{aligned}
\nabla_{\mathbf{r}}\left[\nabla_{\mathbf{r}}^{2} n(r)\right] & =\hat{\mathbf{r}}\left[n^{\prime \prime \prime}(r)+\frac{2}{r} n^{\prime \prime}(r)-\frac{2}{r^{2}} n^{\prime}(r)\right] \\
& =12 \int\left(\nabla_{\mathbf{r}} \Psi\right)\left(\nabla_{\mathbf{r}}^{2} \Psi\right) d \mathbf{r}_{2}+4 \int \Psi \nabla_{\mathbf{r}}\left(\nabla_{\mathbf{r}}^{2} \Psi\right) d \mathbf{r}_{2},
\end{aligned}
$$

which is essentially equivalent to the derivative of Eq. (11) with respect to $r$ when the right-hand side of that equation is written as a constant times the Laplacian of the density $n(r)$, the latter step following from Eq. (4) [see also Eq. (15) below]. In Eq. (13), $\hat{\mathbf{r}}$ is defined as the unit vector $\mathbf{r} / r$. Substituting now the separable form in Eq. (2) of the total wave function $\Psi$ for the harmonic confinement models into the general Eq. (13), one calculates, in agreement with Eq. (1), that $n(r)$ is a functional of $\Psi_{R}$. Hence from Eq. (10) or Eq. (11), the kinetic-energy density difference $t_{L}(r)-t_{g}(r)$, which is one focal point below, is a functional of $\Psi_{R}$. But $t_{g}$ and $t_{L}$ are defined from the density matrix (8) by

$$
\begin{gathered}
t_{g}(r)=\left.\frac{\hbar^{2}}{2 m} \frac{\partial^{2}}{\partial \mathbf{r}_{1} \cdot \partial \mathbf{r}_{2}} \gamma\left(\mathbf{r}_{1}, \mathbf{r}_{2}\right)\right|_{\mathbf{r}_{1}=\mathbf{r}_{2}=\mathbf{r}} \\
t_{L}(r)=-\left.\frac{\hbar^{2}}{2 m} \frac{\partial^{2}}{\partial \mathbf{r}_{1}^{2}} \gamma\left(\mathbf{r}_{1}, \mathbf{r}_{2}\right)\right|_{\mathbf{r}_{1}=\mathbf{r}_{2}=\mathbf{r}}
\end{gathered}
$$

It follows from the above relations that we can write

$$
\gamma\left(\mathbf{r}_{1}, \mathbf{r}_{2}\right)=\Gamma_{f}\left[f(G), \Psi_{C} ; \mathbf{r}_{1}, \mathbf{r}_{2}\right]=\gamma\left[n, \Psi_{C} ; \mathbf{r}_{1}, \mathbf{r}_{2}\right]
$$

where $\Gamma_{f}$ and $\gamma$ are now to be regarded as functionals characterizing the whole family of harmonically confined twoelectron models. One could therefore construct the energy variational principle based on $\gamma(\mathbf{b}, \mathbf{c})$ and the pair function $\left[\Psi\left(\mathbf{r}_{1}, \mathbf{r}_{2}\right)\right]^{2}$ from Eq. (2) since $\Psi_{R}(r)$ is determined from relation (5) by $f(G)$ and, hence, using Eq. (4), by the diagonal ground-state density $n(r)$. This in fact turns out not to be necessary for this family of model atoms since essentially the Euler-Lagrange equation of the variational principle is already implicit in the Schrödinger equation (6) for $\Psi_{R}(r)$, when the effective potential given in relation (7) is employed.

Below we illustrate the functional forms in Eq. (15) more compactly by calculating what amounts to the derivatives of $\gamma$ in Eq. (14). Explicitly, we shall first obtain the difference $t_{L}(r)-t_{g}(r)$ set out in Eq. (10). But Eq. (1) already gives $n(r)$ itself as a functional of $\Psi_{R}$ and, hence, Eq. (10) is equivalent to

$$
t_{L}(r)-t_{g}(r)=T\left[\Psi_{R} ; a, r\right]
$$

Inserting Eq. (1) into Eq. (10) yields then, after a short calculation, 


$$
\begin{aligned}
T(r)= & \frac{2 \hbar^{2}}{a^{3} m \pi^{1 / 2}} \frac{e^{-r^{2} / a^{2}}}{r} \int_{0}^{\infty} d y y \\
& \times\left\{4 r(a y) \cosh \left(\frac{r y}{a}\right)-\left[4 r^{2}+a^{2}\left(y^{2}-2\right)\right] \sinh \left(\frac{r y}{a}\right)\right\} \\
& \times \exp \left(-\frac{y^{2}}{4}\right)\left[\Psi_{R}(a y)\right]^{2} .
\end{aligned}
$$

To illustrate one form of the kinetic-energy density, we appeal next to the result given in [4], namely,

$$
t(r)=\frac{\hbar^{2}}{4 m}\left[\nabla_{\mathbf{r}} \Psi_{C}(r)\right]^{2}+\frac{\hbar^{2}}{m}\left[\nabla_{\mathbf{r}} \Psi_{R}(r)\right]^{2}
$$

For the one model discussed in Appendix B, a numerical comparison of this result (18) for kinetic-energy density $t(r)$ will be made with $t_{g}(r)$ from Eq. (14).

\section{Explicit form of relative motion probability density $P(r)$ in terms of scattering factor $f(G)$}

To illustrate the functional relation $\Gamma_{f}$ for the density matrix $\gamma$ written formally in Eq. (15) for the present family, let us first rewrite Eq. (5) in the form

$$
f(G)=2 \exp \left(-\frac{G^{2} a^{2}}{4}\right) \int \Psi_{R}^{2}(r) \exp \left(i \frac{\mathbf{G} \cdot \mathbf{r}}{2}\right) d \mathbf{r} .
$$

Then by Fourier inversion, we have

$$
\Psi_{R}^{2}(r) \equiv P(r)=\frac{1}{16 \pi^{3}} \int f(G) \exp \left(\frac{G^{2} a^{2}}{4}\right) \exp \left(-i \frac{\mathbf{G} \cdot \mathbf{r}}{2}\right) d \mathbf{G} .
$$

Below in Sec. III, we shall use this result (20) in the ff interaction term, entering the differential virial theorem (DVT) to which we now turn.

\section{ALTERNATIVE ROUTE FOR INTRODUCTION OF INTERACTION $u\left(r_{12}\right)$ VIA THE DVT}

At this stage, let us introduce the interaction $u\left(r_{12}\right)$ by an alternative route starting from the differential virial theorem established in [8]. This reads as

$$
-n(r) \frac{\partial V(r)}{\partial r}=-\frac{n^{\prime \prime \prime}(r)}{4}-\frac{n^{\prime \prime}(r)}{2 r}+\frac{n^{\prime}(r)}{2 r^{2}}+\hat{\mathbf{r}} \cdot \mathbf{z}(\mathbf{r})+\hat{\mathbf{r}} \cdot \mathbf{f}_{f f}(\mathbf{r}),
$$

where the interfermion force $\mathbf{f}_{f f}(\mathbf{r})=\int \nabla_{\mathbf{r}} u\left(\left|\mathbf{r}-\mathbf{r}_{2}\right|\right) \Gamma\left(\mathbf{r r}_{2}\right) d \mathbf{r}_{2}$, while $\Gamma$ denotes the pair-correlation function.

We define immediately below the kinetic-energy density tensor $t_{\alpha \beta}(\mathbf{r})$ quite generally from the 1DM (first-order density matrix) as [8]

$$
t_{\alpha \beta}(\mathbf{r})=\frac{\hbar^{2}}{4 m}\left[\frac{\partial^{2}}{\partial r_{\alpha}^{\prime} \partial r_{\beta}^{\prime \prime}} \gamma\left(\mathbf{r}^{\prime}, \mathbf{r}^{\prime \prime}\right)+\frac{\partial^{2}}{\partial r_{\beta}^{\prime} \partial r_{\alpha}^{\prime \prime}} \gamma\left(\mathbf{r}^{\prime}, \mathbf{r}^{\prime \prime}\right)\right]_{\mathbf{r}^{\prime \prime}=\mathbf{r}^{\prime}=\mathbf{r}} .
$$

Then, inserting the known result (8) for $\gamma$ in Eq. (22) for the present class of models, it is straightforward though lengthy (Sec. VI) to obtain the shape of $t_{\alpha \beta}(\mathbf{r})$ as

$$
t_{\alpha \beta}(\mathbf{r})=\frac{r_{\alpha} r_{\beta}}{r^{2}} t_{1}(r)+\delta_{\alpha \beta} t_{2}(r)
$$

The precise form of $t_{1}(r)$ is rather detailed so we first note that the trace of $t_{\alpha \beta}(r)$, giving the (gradient) kinetic-energy density $t_{g}(r)$ in Eq. (14) is evidently

$$
t_{g}(r)=t_{1}(r)+3 t_{2}(r) .
$$

We record the simpler form of $t_{2}(r)$ for the example of the Moshinsky atom [9] in Appendix A [Eq. (A12)]. The scalar quantity $\hat{\mathbf{r}} \cdot \mathbf{z}(\mathbf{r})$ appearing in Eq. (21) is defined by Holas and March [8], $\mathbf{z}(\mathbf{r})$ having component $z_{\alpha}(\mathbf{r})$ given by

$$
z_{\alpha}(\mathbf{r})=2 \sum_{\beta} \frac{\partial t_{\alpha \beta}(\mathbf{r})}{\partial r_{\beta}},
$$

with $t_{\alpha \beta}(\mathbf{r})$ as in Eq. (23). Of course, $t_{1}(r)$ and $t_{2}(r)$ in that equation depend again on the relative motion wave function $\Psi_{R}(r)$ as this characterizes the 1DM in Eq. (8), from which $\mathbf{z}(\mathbf{r})$ via Eq. (25) is obtained. Given $t_{1}(r)$ and $t_{2}(r)$, the magnitude $z(r)$ of the vector field $\mathbf{z}(\mathbf{r})$ can be readily found from Eqs. (23) and (25) as

$$
z(r)=4\left[\frac{t_{1}(r)}{r}+\frac{1}{2} t_{1}^{\prime}(r)+\frac{1}{2} t_{2}^{\prime}(r)\right] .
$$

Hence, the main outcome of this section can be expressed via Eqs. (21) and (26) as the differential equation

$$
\begin{aligned}
& \frac{n^{\prime \prime \prime}(r)}{4}+\frac{n^{\prime \prime}(r)}{2 r}-\frac{n^{\prime}(r)}{2 r^{2}}-n(r) \frac{\partial V(r)}{\partial r} \\
& =\hat{\mathbf{r}} \cdot \int \Psi^{2}\left(\mathbf{r}, \mathbf{r}_{2}\right) \nabla_{\mathbf{r}} u\left(\left|\mathbf{r}-\mathbf{r}_{2}\right|\right) d \mathbf{r}_{2}+z(r) .
\end{aligned}
$$

Naturally, the last two terms are again functionals of the relative motion wave function $\Psi_{R}(r)$. The term involving $\boldsymbol{\nabla}_{\mathbf{r}} u$ is, using Eq. (2), given by $\int \Psi_{C}^{2}\left(\frac{\left|\mathbf{r}+\mathbf{r}_{2}\right|}{2}\right) P\left(\left|\mathbf{r}-\mathbf{r}_{2}\right|\right)\left[\boldsymbol{\nabla}_{\mathbf{r}} u(\mid \mathbf{r}\right.$ $\left.\left.-\mathbf{r}_{2} \mid\right)\right] d \mathbf{r}_{2}$ with $P(r)=\Psi_{R}^{2}$ and as already emphasized from Eqs. (5) and (20), $P$ can be replaced by the Fourier transform of $f(G) \exp \left(\frac{G^{2} a^{2}}{4}\right)$. To be quite specific we have, using Eq. (20),

$$
\begin{aligned}
\hat{\mathbf{r}} \cdot \int & \Psi_{C}^{2}\left(\frac{\left|\mathbf{r}+\mathbf{r}_{2}\right|}{2}\right) P\left(\left|\mathbf{r}-\mathbf{r}_{2}\right|\right)\left[\nabla_{\mathbf{r}} u\left(\left|\mathbf{r}-\mathbf{r}_{2}\right|\right)\right] d \mathbf{r}_{2} \\
= & \hat{\mathbf{r}} \cdot \int\left[\nabla_{\mathbf{r}} u\left(\left|\mathbf{r}-\mathbf{r}_{2}\right|\right)\right] \Psi_{C}^{2}\left(\frac{\left|\mathbf{r}+\mathbf{r}_{2}\right|}{2}\right) \int f(G) \\
& \times \exp \left(\frac{G^{2} a^{2}}{4}\right) \exp \left(-i \frac{\mathbf{G} \cdot\left(\mathbf{r}-\mathbf{r}_{2}\right)}{2}\right) d \mathbf{G} d \mathbf{r}_{2} .
\end{aligned}
$$

Assuming that we can interchange the order of integration in Eq. (28), the above term, for a given $u\left(\left|\mathbf{r}-\mathbf{r}_{2}\right|\right)$ can be written as $(\mathcal{F}$ denotes Fourier transform)

$$
\hat{\mathbf{r}} \cdot \int f(G) \exp \left(\frac{G^{2} a^{2}}{4}\right) \mathcal{F}\left\{\left[\nabla_{\mathbf{r}} u\left(\left|\mathbf{r}-\mathbf{r}_{2}\right|\right)\right] \Psi_{C}^{2}\left(\frac{\left|\mathbf{r}+\mathbf{r}_{2}\right|}{2}\right)\right\} d \mathbf{G},
$$

where $\Psi_{C}$ is known explicitly from Eq. (9). We shall illustrate Eqs. (28) and (29) for the Moshinsky atom in Appendix 
A. This makes it clear that Eq. (27) is now an integrodifferential equation to be solved for the ground-state density, after inputting a specific pair interaction $u\left(r_{12}\right)$ and the harmonic confinement potential $V(r)=\frac{1}{2} m \omega^{2} r^{2}$.

\section{INDEPENDENT-PARTICLE LIMIT OF THE INTEGRO- DIFFERENTIAL EQ. (27)}

Due to the relative complexity of the integro-differential (27), let us consider next the simplification obtained when we set the interaction $u$ equal to zero. Then $\mathbf{z}(\mathbf{r})$ becomes its single-particle counterpart $\mathbf{z}_{s}$. This is readily derived from the idempotent Dirac density matrix $\gamma\left(\mathbf{r}_{1}, \mathbf{r}_{2}\right)$ given by

$$
\gamma\left(\mathbf{r}_{1}, \mathbf{r}_{2}\right)=\left[n\left(r_{1}\right)\right]^{1 / 2}\left[n\left(r_{2}\right)\right]^{1 / 2},
$$

after insertion in Eq. (22). From Eqs. (22), (25), and (30), it is found after a short calculation that $t_{1}$ and $t_{2}$ in Eq. (23) are

$$
\begin{gathered}
t_{1}=\left(\hbar^{2} / 8 m\right) \frac{n^{\prime 2}(r)}{n}, \\
t_{2}=0,
\end{gathered}
$$

and the magnitude $z_{s}(r)$ of the single-particle vector field $\mathbf{z}_{s}(r)$ is then obtained as

$$
\begin{aligned}
z_{s}(r) & =\frac{\hbar^{2}}{2 m} \frac{n^{\prime}(r)}{n}\left[\frac{n^{\prime}(r)}{r}-\frac{n^{\prime 2}(r)}{2 n(r)}+n^{\prime \prime}(r)\right] \\
& =4\left[\frac{t_{w}(r)}{r}+\frac{1}{2} \frac{\partial t_{w}(r)}{\partial r}\right] .
\end{aligned}
$$

Here the von Weizsäcker kinetic-energy density $t_{w}(r)$ is given by $\left(\hbar^{2} / 8 m\right)\left(n^{\prime 2} / n\right)$ and is therefore equal to $t_{1}(r)$ in this limit. Hence, we find from Eq. (27) with $u(r)=0$, by insertion of $z_{s}(r)$ from Eq. (33), a third-order nonlinear differential equation for $n(r)$. Of course, we already know that this must have as a "solution," the well-known singleparticle equation of von Weizsäcker [10] and this is readily confirmed.

Returning to the family with interaction $u(r)$, the simplest case to treat is the Moshinsky atom [9] with $u\left(r_{12}\right)=\frac{1}{2} K r_{12}^{2}$, and this is worked out in detail in Appendix A. When $K$ is put to zero, Eq. (33) is readily verified in this model, from the results given in that appendix.

\section{GRADIENT KINETIC-ENERGY DENSITY}

We first derive $t_{g}(r)$ defined from the wave function (2) and leave the deduction of $t_{\alpha \beta}$ in Eq. (23) for next section. The gradient kinetic energy $t_{g}(r)$ from the wave function (2) is given by

$$
t_{g}(r)=\frac{\hbar^{2}}{m} \int\left\{\boldsymbol{\nabla}_{\mathbf{r}} \Psi\left(\mathbf{r}, \mathbf{r}_{2}\right)\right\}^{2} d \mathbf{r}_{2} .
$$

Using the explicit form (9) for the center-of-mass wave function $\Psi_{C}$ in Eq. (2), we can calculate $\nabla_{\mathbf{r}} \Psi$ entering in Eq. (34) as

$$
\begin{aligned}
\boldsymbol{\nabla}_{\mathbf{r}} \Psi\left(\mathbf{r}, \mathbf{r}_{2}\right)= & \frac{1}{a^{3 / 2} \pi^{3 / 4}} \\
& \times\left[\frac{\mathbf{r}-\mathbf{r}_{2}}{\left|\mathbf{r}-\mathbf{r}_{2}\right|} \Psi_{R}^{\prime}\left(\left|\mathbf{r}-\mathbf{r}_{2}\right|\right)-\frac{\mathbf{r}+\mathbf{r}_{2}}{4 a^{2}} \Psi_{R}\left(\left|\mathbf{r}-\mathbf{r}_{2}\right|\right)\right] \\
& \times \exp \left(-\frac{\left|\mathbf{r}+\mathbf{r}_{2}\right|^{2}}{8 a^{2}}\right)
\end{aligned}
$$

It is a straightforward, if somewhat complicated matter, to calculate $t_{g}(r)$ by squaring Eq. (35) and inserting into Eq. (34). The desired result for $t_{g}(r)$ emerges then in the functional form

$$
t_{g}(r)=t_{g}\left[\Psi_{R}, \Psi_{R}^{\prime} ; a\right] .
$$

One next completes the angular integration to remove the scalar product $\hat{\mathbf{r}} \cdot \hat{\mathbf{r}}_{2}$ entering the square of Eq. (35), leaving simply a radial integration over $\mathbf{r}_{2}$. This yields the explicit form of the functional in Eq. (36) as

$$
\begin{aligned}
t_{g}(r)= & \frac{2 \hbar^{2}}{a^{3} \pi^{1 / 2} m} \frac{e^{-r^{2} / a^{2}}}{r} \int_{0}^{\infty}\left\{2 a^{2} s \sinh \left(r s / a^{2}\right) \Psi_{R}^{\prime 2}(s)\right. \\
& +\left[\left(s^{2}+2 a^{2}\right) \sinh \left(r s / a^{2}\right)-2 r s \cosh \left(r s / a^{2}\right)\right] \\
& \times \Psi_{R}(s) \Psi_{R}^{\prime}(s)+\frac{s}{8 a^{2}}\left[\left(s^{2}+4 a^{2}+4 r^{2}\right) \sinh \left(r s / a^{2}\right)\right. \\
& \left.\left.-4 r s \cosh \left(r s / a^{2}\right)\right] \Psi_{R}^{2}(s)\right\} e^{-s^{2} / 4 a^{2}} d s .
\end{aligned}
$$

But Eq. (17) allows the Laplacian form $t_{L}(r)$ of kineticenergy density to be obtained as

$$
t_{L}(r)=t_{g}(r)-\frac{2 \hbar^{2}}{a^{3} m \pi^{1 / 2}} \frac{e^{-r^{2} / a^{2}}}{r} \int_{0}^{\infty} d y \tau(y, r, a)\left[\Psi_{R}(a y)\right]^{2},
$$

where $\tau(y, r, a)$ is

$$
\begin{aligned}
& \tau(y, r, a)=y\left\{4 r(a y) \cosh \left(\frac{r y}{a}\right)\right. \\
& \left.-\left[4 r^{2}+a^{2}\left(y^{2}-2\right)\right] \sinh \left(\frac{r y}{a}\right)\right\} \exp \left(-\frac{y^{2}}{4}\right) \text {. }
\end{aligned}
$$

\section{MAGNITUDE $z(r)$ OF VECTOR FIELD ENTERING DIFFERENTIAL VIRIAL THEOREM (21) IN TERMS OF RELATIVE MOTION WAVE FUNCTION $\Psi_{R}$}

As mentioned briefly above, we use the density matrix (8), plus $\Psi_{C}(r)$ again from Eq. (9), to derive the kineticenergy density tensor $t_{\alpha \beta}$ defined in Eq. (22). Hence, we extract the key quantities $t_{1}(r)$ and $t_{2}(r)$ entering the form of $t_{\alpha \beta}$ in Eq. (23).

As this is a straightforward but tiresome derivation, we give here the final result for $t_{\alpha \beta}$ as 


$$
\begin{aligned}
t_{\alpha \beta}(r)= & \left\{\frac{2 \hbar^{2}}{a^{3} \pi^{1 / 2} m} e^{-\left(r^{2}\right) / a^{2}} \int_{o}^{\infty}-\left[\frac{3}{4 a^{2}} P(x)+\frac{1}{2 x} P^{\prime}(x)+\frac{a^{4}}{r^{2}} g(x)\right] \frac{a^{2} x}{r} \sinh \left(\frac{x r}{a^{2}}\right)+\frac{x^{2} a^{4}}{r^{2}} g(x) \cosh \left(\frac{x r}{a^{2}}\right) d x\right\} \delta_{\alpha \beta} \\
& +\left\{\frac{2 \hbar^{2}}{a^{3} \pi^{1 / 2} m} e^{-\left(r^{2}\right) / a^{2}} \int_{0}^{\infty} 2 P(x)\left[\left(\frac{x}{r}+\frac{x r}{a^{2}}\right) \sinh \left(\frac{x r}{a^{2}}\right)-\frac{x^{2}}{a^{2}} \cosh \left(\frac{x r}{a^{2}}\right)\right]\right. \\
& \left.+a^{4} x g(x)\left[\left(\frac{3 a^{2}}{r^{3}}+\frac{x^{2}}{a^{2} r}\right) \sinh \left(\frac{x r}{a^{2}}\right)-\frac{3 x}{r^{2}} \cosh \left(\frac{x r}{a^{2}}\right)\right] d x\right\} \frac{r_{\alpha} r_{\beta}}{r^{2}}
\end{aligned}
$$

where $P(x)$ is given in Eq. (20) and

$$
g(x)=\frac{2}{a^{4}} P(x)+\frac{1}{2 x^{3}} P^{\prime}(x)+\frac{1}{2 x^{2}} \frac{P^{\prime 2}(x)}{P(x)}-\frac{1}{2 x^{2}} P^{\prime \prime}(x) .
$$

Therefore, $t_{1}(r)$ and $t_{2}(r)$ are

$$
\begin{aligned}
t_{1}(r)= & \frac{2 \hbar^{2}}{a^{3} \pi^{1 / 2} m} e^{-\left(r^{2}\right) / a^{2}} \int_{o}^{\infty} 2 P(x) \\
& \times\left[\left(\frac{x}{r}+\frac{x r}{a^{2}}\right) \sinh \left(\frac{x r}{a^{2}}\right)-\frac{x^{2}}{a^{2}} \cosh \left(\frac{x r}{a^{2}}\right)\right]+a^{4} x g(x) \\
& \times\left[\left(\frac{3 a^{2}}{r^{3}}+\frac{x^{2}}{a^{2} r}\right) \sinh \left(\frac{x r}{a^{2}}\right)-\frac{3 x}{r^{2}} \cosh \left(\frac{x r}{a^{2}}\right)\right] d x,(4) \\
t_{2}(r)= & \frac{2 \hbar^{2}}{a^{3} \pi^{1 / 2} m} e^{-r^{2} / a^{2}} \int_{o}^{\infty}-\left[\frac{3}{4 a^{2}} P(x)+\frac{1}{2 x} P^{\prime}(x)\right. \\
& \left.+\frac{a^{4}}{r^{2}} g(x)\right] \frac{a^{2} x}{r} \sinh \left(\frac{x r}{a^{2}}\right)+\frac{x^{2} a^{4}}{r^{2}} g(x) \cosh \left(\frac{x r}{a^{2}}\right) d x .
\end{aligned}
$$

Results for $t_{1}(r)$ and $t_{2}(r)$ reduce to the form given in Appendix A for the Moshinsky atom in Eqs. (A13) and (A14). The magnitude of vector field $z(r)$ can then be derived by insertion of Eqs. (42) and (43) into Eq. (26), but since the detail proliferates considerably we shall not give the explicit form of $z(r)$.

\section{SUMMARY AND FUTURE DIRECTIONS}

It is shown here that the exact density-matrix theory given in Eqs. (1) and (8) can be viewed variationally as based on the integro-differential equation (27). What is unique to the family of models explained in the title is that the last two terms in Eq. (27) are calculable explicitly in terms of the probability density $P(r)=\Psi_{R}^{2}(r)$ of the relative motion from the density matrix $\gamma$ in Eq. (8). But Eq. (5) then shows that $P(r)$ is uniquely fixed by the Fourier transform of $f(G) \exp \left(\frac{G^{2} a^{2}}{4}\right), f(G)$ being the x-ray scattering factor defined in Eq. (4) as the Fourier transform of the desired electron density $n(r)$. In the above sense, Eq. (27) has the general shape

$$
\frac{r}{4} \frac{\partial}{\partial r}\left[\nabla^{2} n(r)\right]-r n(r) \frac{\partial V(r)}{\partial r}=Q[u(r) ; f(G), a],
$$

which we have chosen to write with the virial of the force $-\frac{\partial V(r)}{\partial r}$ on the left-hand side. This is plainly of the form of an integro-differential equation to solve for the ground-state density $n(r)$, the length a characterizing the harmonic external potential $V(r)$. In Appendix A, a form of $Q$ in Eq. (44) is displayed which is specific to the Moshinsky atom, and involves $f(G)$ and its derivatives. Further motivation for writing the functional form (44) of $Q$ is provided by the expression (29) for the interparticle force entering Eq. (27).

As to future directions, we wish to emphasize the relevance of parts of the present study for the determination of the ground-state density $n(r, Z)$ of nonrelativistic He-like atomic ions of nuclear charge $Z e$. This problem has remained unsolved since the discovery of Schrödinger wave mechanics some 8 decades ago. So we conclude by emphasizing the equations in the present paper, which immediately apply to such He-like ions. The first of these is Eq. (11) relating kinetic-energy density difference to the x-ray scattering factor $f(G)$, which incidentally was measured in early work on He vapor. Equation (13) is also applicable to $\mathrm{He}$, as is its expanded form in Appendix $\mathrm{C}$, which now contains also the ground-state energy $E$, again known from experiment. The final equation we single out for its generality and, in particular, its applicability to He, is the differential virial theorem in Eq. (21). However, to date, without some input from experiment these general nonrelativistic equations can be worked out for He-like atomic ions only in the limit of large atomic number $Z$ motivated by the pioneering study of Schwartz [11] on the ground-state electron density $n(r, Z)$ in this (nonrelativistic) large $Z$ limit. In that limit, Gál et al. [12] derived the third-order linear homogeneous differential equation satisfied by the Schwartz density, which has therefore a shape quite reminiscent of the DVT in Eq. (21).

\section{ACKNOWLEDGMENTS}

N.H.M. wishes to acknowledge that his contribution to the present study was brought to fruition during a stay at DIPC, San Sebastián. N.H.M. also thanks Professor P. M. Etxenique for generous hospitality. We acknowledge funding by the Spanish Ministry of Education (FIS2007-65702-C0201), "Grupos Consolidados UPV/EHU del Gobierno Vasco" 
(IT-319-07) the European Community through e-0I3 ETSF project (Contract Number 211956).

APPENDIX A: EXPLICIT FORM OF $\hat{\mathbf{r}} \cdot \mathbf{z}(\mathbf{r})+\hat{\mathbf{r}} \cdot \mathbf{f}_{f f}(r)$ APPEARING IN THE DVT EQ. (21) FOR MOSHINSKY ATOM AS WELL AS THE KINETIC-ENERGY DENSITY TENSOR

First, we remind that in Appendixes A and B, $\hat{\mathbf{r}} \cdot \mathbf{z}(\mathbf{r})+\hat{\mathbf{r}} \cdot \mathbf{f}_{f f}(r)$ has been calculated from Eq. (21) and not through their definitions. Hence, it must not be used in the equation since it yields nothing but tautology.

As one of our aims, this appendix gives the explicit forms of the quantity $\hat{\mathbf{r}} \cdot \mathbf{z}(\mathbf{r})+\hat{\mathbf{r}} \cdot \mathbf{f}_{f f}(r)$ appearing in DVT Eq. (21) for the totally harmonic Moshinsky model. The known ground-state density for harmonic confinement potential $V(r)=\frac{1}{2} r^{2}$ and interparticle interaction $u\left(r_{12}\right)=\frac{1}{2} K r_{12}^{2}$ is

$$
n(r)=\frac{2 \beta^{3 / 2}}{\pi^{3 / 2}} \exp \left(-\beta r^{2}\right)
$$

where $\beta=\frac{2 \alpha-1}{\alpha}$ while $\alpha$ is given by

$$
\alpha=\frac{1}{2}\left[(1+2 K)^{1 / 2}+1\right] .
$$

To compare with the DVT Eq. (21), we need the third-order differential equation satisfied by $n(r)$ in Eq. (A1). This is readily verified to be

$$
r^{2} n^{\prime \prime \prime}+2 r n^{\prime \prime}-\left[4 \beta^{2} r^{4}-6 \beta r^{2}+2\right] n^{\prime}-8 \beta^{2} r^{3} n=0 .
$$

Using Eq. (27) with $V(r)=\frac{1}{2} r^{2}$, the right-hand side is simply $\hat{\mathbf{r}} \cdot \mathbf{z}(\mathbf{r})+\hat{\mathbf{r}} \cdot \mathbf{f}_{f f}(r)$. Hence, one finds the exact expression

$$
\hat{\mathbf{r}} \cdot \mathbf{z}(\mathbf{r})+\hat{\mathbf{r}} \cdot \mathbf{f}_{f f}(r)=\left[\beta^{2} r^{2}-\frac{3}{2} \beta\right] n^{\prime}(r)+[2 \beta r-r] n(r)
$$

The single-particle limit $z_{s}(r)$ is readily found from Eq. (A4) by putting $K=0$, when one has the left-hand side of Eq. (A4) simply as $z_{s}(r)$, while the right-hand side in the same limit has $\alpha=1$ from Eq. (A2) and hence $\beta=1$. Thus, for this harmonic case

$$
z_{s}(r)=\left(r^{2}-\frac{3}{2}\right) n^{\prime}(r)+r n(r) .
$$

Using again Eq. (A1) in the von Weizsäcker form $t_{w}=\left(\hbar^{2} / 8 m\right)\left(n^{\prime 2} / n\right)$, we find $t_{w}=(1 / 2) \beta^{2} r^{2} n$ and, hence,

$$
z_{s}(r)=\left(4 r-2 r^{3}\right) n(r) .
$$

This is equivalent to Eq. (A5) when we use the explicit form (A1) of $n(r)$ for which $n^{\prime}(r)=-2 \beta r n$ with $\beta=1$ in the $K \rightarrow 0$ limit.

Using the explicit form of $\gamma\left(\mathbf{r}, \mathbf{r}^{\prime}\right)$ in terms of $n(r)$ given in $[13]$

$$
\begin{aligned}
\gamma\left(\mathbf{r}, \mathbf{r}^{\prime}\right)= & \left(\frac{2 \beta^{3 / 2}}{\pi^{3 / 2}}\right)^{\alpha^{2} / 4 \alpha-2}\left\{n\left[\left(r^{2}+r^{\prime 2}\right)^{1 / 2}\right]\right\}^{\alpha^{2} / 4 \alpha-2} \\
& \times\left\{n\left(\frac{\left(r^{2}+r^{\prime 2}+2 \mathbf{r} \cdot \mathbf{r}^{\prime}\right)^{1 / 2}}{2}\right)\right\}^{-\left[(\alpha-1)^{2}\right] / 2 \alpha-1},
\end{aligned}
$$

we have obtained the element $t_{12}(\mathbf{r})$ of the kinetic-energy density tensor $t_{\alpha \beta}(\mathbf{r})$ in Eq. (22) as

$$
\begin{aligned}
t_{12}(\mathbf{r})= & \frac{\hbar^{2}}{16 m(2 \alpha-1)^{2}}\left(\frac{2 \beta^{3 / 2}}{\pi^{3 / 2}}\right)^{\alpha^{2} / 4 \alpha-2} \frac{x y}{r^{4}}\{n(r)\}^{[1-\alpha(\alpha+2)] / 2 \alpha-1}\{n(\sqrt{2} r)\}^{\left\{\left(\alpha^{2}\right) /(4 \alpha-2)\right\}-2} \\
& \times\left[2 r^{2}(\alpha-1)^{2} \alpha^{2} n^{2}(\sqrt{2} r) n^{\prime 2}(r)+2(\alpha-1)^{2} n(r) n(\sqrt{2} r)\left[\sqrt{2} r^{2} \alpha^{2} n^{\prime}(r) n^{\prime}(\sqrt{2} r)\right]-(2 \alpha-1) n(\sqrt{2} r)\left[r n^{\prime}(r)-r^{2} n^{\prime \prime}(r)\right]\right. \\
& \left.+\alpha^{2} n^{2}(r)\left\{r^{2}[\alpha(\alpha-4)+2] n^{\prime 2}(\sqrt{2} r)-(2 \alpha-1) n(\sqrt{2})\left[\sqrt{2} r n^{\prime}(\sqrt{2} r)-2 r^{2} n^{\prime \prime}(\sqrt{2})\right]\right\}\right] .
\end{aligned}
$$

In the independent-particle limit $K \rightarrow 0$, or equivalently $\alpha \rightarrow 1$, Eq. (A8) becomes

$$
t_{12}(\mathbf{r})=\left(\frac{2}{\pi^{3 / 2}}\right)^{1 / 2} \frac{\hbar^{2}}{16 m n^{3 / 2}(\sqrt{2} r)} \frac{x y}{r^{3}}\left\{r n^{\prime 2}(\sqrt{2} r)+n(\sqrt{2} r)\left[\sqrt{2} n^{\prime}(\sqrt{2} r)-2 r n^{\prime \prime}(\sqrt{2} r)\right]\right\} .
$$

Thus, we confirm the shape of $t_{\alpha \beta}(\mathbf{r})$ in Eq. (23) and find for the Moshinsky model the form of $t_{1}(r)$ to be

$$
\begin{aligned}
t_{1}(r)= & \frac{\hbar^{2}}{16 m(2 \alpha-1)^{2} r^{2}}\left(\frac{2 \beta^{3 / 2}}{\pi^{3 / 2}}\right)^{\alpha^{2} / 4 \alpha-2}\{n(r)\}^{[1-\alpha(\alpha+2)] / 2 \alpha-1}\{n(\sqrt{2} r)\}^{\left[\left(\alpha^{2}\right) /(4 \alpha-2)\right]-2} \\
& \times\left[2 r^{2}(\alpha-1)^{2} \alpha^{2} n^{2}(\sqrt{2} r) n^{\prime 2}(r)+2(\alpha-1)^{2} n(r) n(\sqrt{2} r)\left[\sqrt{2} r^{2} \alpha^{2} n^{\prime}(r) n^{\prime}(\sqrt{2} r)\right]-(2 \alpha-1) n(\sqrt{2} r)\left[r n^{\prime}(r)-r^{2} n^{\prime \prime}(r)\right]+\alpha^{2} n^{2}(r)\right. \\
& \left.\times\left\{r^{2}[\alpha(\alpha-4)+2] n^{\prime 2}(\sqrt{2} r)-(2 \alpha-1) n(\sqrt{2})\left[\sqrt{2} r n^{\prime}(\sqrt{2} r)-2 r^{2} n^{\prime \prime}(\sqrt{2})\right]\right\}\right] .
\end{aligned}
$$

This has the independent-particle limit when $\alpha \rightarrow 1$ given by

$$
t_{1}(r)=\left(\frac{2}{\pi^{3 / 2}}\right)^{1 / 2} \frac{\hbar^{2}}{16 m r n^{3 / 2}(\sqrt{2} r)}\left\{r n^{\prime 2}(\sqrt{2} r)+n(\sqrt{2} r)\left[\sqrt{2} n^{\prime}(\sqrt{2} r)-2 r n^{\prime \prime}(\sqrt{2} r)\right]\right\} .
$$

Similarly, we have obtained $t_{2}(r)$ in Eq. (23) as 


$$
t_{2}(r)=-\left(\frac{2 \beta^{3 / 2}}{\pi^{3 / 2}}\right)^{\alpha^{2} / 4 \alpha-2} \frac{\hbar^{2}(\alpha-1)^{2}}{8 m(2 \alpha-1) r}\{n(r)\}^{\alpha^{2} / 1-2 \alpha}\{n(\sqrt{2} r)\}^{\alpha^{2} / 4 \alpha-2} n^{\prime}(r),
$$

which tends to zero in the independent-particle limit $\alpha \rightarrow 1$. Hence, it follows from Eq. (24) that $t_{1}(r)$ in Eq. (A11) is equal to the gradient form $t_{g}(r)$ of the kinetic-energy density in Eq. (24). Using the explicit ground-state electron density, it is readily verified that Eq. (A11) gives back the von Weizsäcker form $t_{g}(r)=t_{w}(r)=\left(\hbar^{2} / 8 m\right)\left[n^{\prime 2}(r) / n(r)\right]$.

If we use the form of the density in Eq. (A1), $t_{1}(r)$ and $t_{2}(r)$ simplify to

$$
t_{1}(r)=\frac{\hbar^{2}}{2 m}\left(\frac{2 \alpha-1}{\alpha}\right)^{2} r^{2} n(r),
$$

and

$$
t_{2}(r)=\frac{\hbar^{2}}{4 m} \frac{(\alpha-1)^{2}}{\alpha} n(r)
$$

Inserting Eqs. (A13) and (A14) into Eq. (26) yields $z(r)$, an example of which we have plotted in Fig. 1.

Of course, there is no claim to universality or, for this model, uniqueness of functionals of $n$ displayed in this appendix. Also, the external potential has been chosen here for simplicity as $V(r)=(1 / 2) r^{2}$, and for other choices the length $a$ characterizing the harmonic external potential $V(r)$ in general will also appear in such functionals of the ground-state density.

To conclude this appendix, we exemplify Eq. (44) of the main text by displaying an explicit form of $Q$ entering that equation for the Moshinsky model. It is readily verified from Eq. (A3) that

$$
\frac{r}{4} \frac{\partial}{\partial r}\left[\nabla^{2} n(r)\right]-r n(r) \frac{\partial V(r)}{\partial r}=\left[5 \beta^{2} r^{2}-r^{2}-2 \beta^{3} r^{4}\right] n(r) .
$$

Thus, in atomic units, it results in

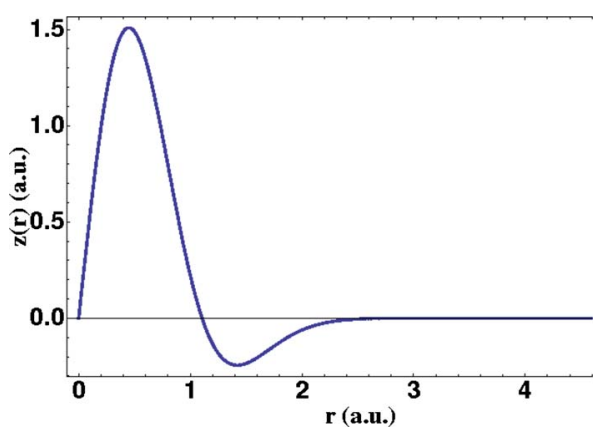

FIG. 1. (Color online) Shows magnitude $z(r)$ of the vector field $\mathbf{z}(\mathbf{r})$ entering the DVT Eq. (21) for the Moshinsky atom for parameter $\beta=3 / 2$ measuring the interparticle interaction strength.

$$
Q=\left[5 \beta^{2} r^{2}-r^{2}-2 \beta^{3} r^{4}\right] n(r) .
$$

But in terms of the x-ray scattering factor $f(G)$ defined in terms of the density $n(r)$ in Eq. (4)

$$
\nabla_{\mathbf{G}}^{2} f(G)=-\int r^{2} n(r) \exp (i \mathbf{G} \cdot \mathbf{r}) d \mathbf{r},
$$

while

$$
\nabla_{\mathbf{G}}^{2}\left[\nabla_{\mathbf{G}}^{2} f(G)\right]=\int r^{4} n(r) \exp (i \mathbf{G} \cdot \mathbf{r}) d \mathbf{r} .
$$

Hence, for this example, using the Fourier inversion of Eqs. (A17) and (A18), we have $Q$ explicitly in terms of derivatives of $f(G)$ and the pair interaction $u(r)=(1 / 2) K r^{2}$ since $\beta$ entering Eq. (A16) characterizes the strength $K$ of $u(r)$.

\section{APPENDIX B: INVERSE SQUARE-LAW MODEL}

For the inverse square-law model, the wave function of Crandall et al. [14] reads as

$$
\begin{aligned}
\Psi\left(\mathbf{r}, \mathbf{r}_{2}\right)= & {\left[4 \pi \Gamma\left(\frac{3}{2}+\alpha\right) 4^{\alpha+1} a^{3+2 \alpha}\right]^{-1 / 2}\left|\mathbf{r}-\mathbf{r}_{2}\right|^{\alpha} } \\
& \times \exp \left(-m \omega r^{2} / 2 \hbar\right) \exp \left(-m \omega r_{2}^{2} / 2 \hbar\right),
\end{aligned}
$$

where $u\left(r_{12}\right)=\lambda / r_{12}^{2}, \alpha$ being related to the strength $\lambda$ by $\left[\left(1+4 \lambda m / \hbar^{2}\right)^{1 / 2}-1\right] / 2$. For general interaction strength $\alpha$, the differential equation for the density in [15] has the form

$$
\frac{\hbar}{4 m \omega} r n^{\prime \prime}(r)+\left(\frac{\hbar}{2 m \omega}+\frac{3}{2} r^{2}\right) n^{\prime}(r)+r\left(\frac{3}{2}-\alpha+\frac{2 m \omega}{\hbar} r^{2}\right) n(r)
$$

$=0$.

Inserting into the DVT Eq. (21), we obtain an explicit expression in terms of $n$ and its derivatives for $\hat{\mathbf{r}} \cdot \mathbf{z}(\mathbf{r})$ $+\hat{\mathbf{r}} \cdot \mathbf{f}_{f f}(r)$, which reads as

$$
\hat{\mathbf{r}} \cdot \mathbf{z}(\mathbf{r})+f_{f f}(r)=-n(r) \frac{\partial V(r)}{\partial r}+\frac{n^{\prime \prime}(r)}{2 r}-\frac{n^{\prime}(r)}{2 r^{2}}+\frac{1}{4} n^{\prime \prime \prime}(r) .
$$

Multiplying Eq. (B2) throughout by $\frac{m \omega}{\hbar r}$, we readily obtain

$$
\frac{n^{\prime \prime}(r)}{4}+\left(\frac{1}{2 r}+\frac{3}{2} \frac{m \omega r}{\hbar}\right) n^{\prime}(r)+\frac{m \omega}{\hbar}\left(\frac{3}{2}-\alpha+\frac{2 m \omega r^{2}}{\hbar}\right) n(r)=0 .
$$


Dividing both sides of Eq. (B3) by $n(r)$ and then differentiating with respect to $r$ yields

$$
\begin{gathered}
4\left(\frac{m \omega}{\hbar}\right)^{2} r+\frac{1}{4}\left(\frac{n n^{\prime \prime \prime}-n^{\prime \prime} n^{\prime}}{n^{2}}\right)+\left(-\frac{1}{2 r^{2}}+\frac{3}{2} \frac{m \omega}{\hbar}\right) \frac{n^{\prime}}{n} \\
+\left(\frac{1}{2 r}+\frac{3}{2} \frac{m \omega}{\hbar} r\right)\left(\frac{n n^{\prime \prime}-n^{\prime 2}}{n^{2}}\right)=0 .
\end{gathered}
$$

Substituting for $n^{\prime \prime \prime}(r)$ in Eq. (B3) using Eq. (B5) results in an equation for $\hat{\mathbf{r}} \cdot \mathbf{z}(\mathbf{r})+\hat{\mathbf{r}} \cdot \mathbf{f}_{f f}(r)$ in terms of $n(r)$ and its derivatives $n^{\prime}(r)$ and $n^{\prime \prime}(r)$ plus $V(r)$. The result reads as

$$
\begin{aligned}
z(r)+\hat{\mathbf{r}} \cdot \mathbf{f}_{f f}(r)= & -m \omega^{2} r n(r)-4\left(\frac{m \omega}{\hbar}\right)^{2} r n(r)+\frac{1}{4} \frac{n^{\prime \prime} n^{\prime}}{n} \\
& -\frac{3}{2} \frac{m \omega}{\hbar} n^{\prime}-\left(\frac{1}{2 r}+\frac{3}{2} \frac{m \omega}{\hbar} r\right)\left(n^{\prime \prime}-\frac{n^{\prime 2}}{n}\right)
\end{aligned}
$$

Equation (B6) is a general expression for the basic $f f$ interaction term in the inverse square-law model, $z(r)$ correcting the single-particle form $z_{s}(r)$ in Eq. (33), which has already the von Weizsäcker inhomogeneity kinetic energy embedded in it. It is surprising that Eq. (B6) does not have any explicit dependency on value of $\alpha$, which has a connection to the strength of the interparticle interaction.

\section{Gradient kinetic-energy density for inverse square-law interparticle interaction}

Let us take as starting point the result of [7] for the wave function [14] in Eq. (B1). Then the gradient kinetic-energy density may be written [7] as

$$
\begin{aligned}
t_{g}(r)= & \frac{m^{2} \omega^{2} r^{2}}{\hbar^{2}} \frac{n(r)}{2}+\alpha^{2} \int \frac{\left(\nabla_{\mathbf{r}}\left|\mathbf{r}-\mathbf{r}_{2}\right|\right)^{2}}{\left|\mathbf{r}-\mathbf{r}_{2}\right|^{2}} \Psi_{C}^{2}\left(\frac{\left|\mathbf{r}+\mathbf{r}_{2}\right|}{2}\right) \\
& \times \Psi_{R}^{2}\left(\left|\mathbf{r}-\mathbf{r}_{2}\right|\right) d \mathbf{r}_{2}-2 \frac{\alpha m \omega r}{\hbar} \int \frac{1}{\left|\mathbf{r}-\mathbf{r}_{2}\right|}\left(\hat{\mathbf{r}} \cdot \nabla_{\mathbf{r}}\left|\mathbf{r}-\mathbf{r}_{2}\right|\right) \\
& \times \Psi_{C}^{2}\left(\frac{\left|\mathbf{r}+\mathbf{r}_{2}\right|}{2}\right) \Psi_{R}^{2}\left(\left|\mathbf{r}-\mathbf{r}_{2}\right|\right) d \mathbf{r}_{2}
\end{aligned}
$$

Inserting the explicit form of $\Psi_{C}(r)$ in Eq. (9) and $\Psi_{R}(r)$ thereby obtained from Eqs. (2) and (B1) one finds

$$
\begin{aligned}
t_{g}(r)= & \frac{2^{-(7 / 2)-\alpha}}{a^{7} \pi^{3 / 2}(1+2 \alpha)}\left[\left(r^{2}-4 a^{2} \alpha\right) F_{1}\left(\frac{1}{2}+\alpha ; \frac{1}{2} ; \frac{r^{2}}{2 a^{2}}\right)\right. \\
& \left.+2 \alpha\left[r^{2}+2 a^{2}(1+\alpha)\right] F_{1}\left(\frac{1}{2}+\alpha ; \frac{3}{2} ; \frac{r^{2}}{2 a^{2}}\right)\right] e^{-r^{2} / a^{2}}
\end{aligned}
$$

where $F_{1}(a ; b ; z)$ is the confluent hypergeometric function of the first kind [16].

Figure 2 makes a numerical comparison, in atomic units, between the radial kinetic-energy density $4 \pi r^{2} t_{g}(r)$ and the

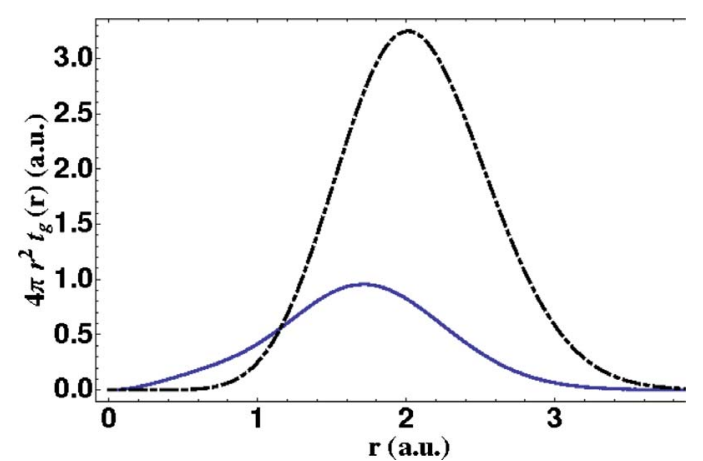

FIG. 2. (Color online) Comparison of correlated and independent-particle energy densities $4 \pi r^{2} t_{g}(r)$ (continuous curve) given analytically in Eq. (B9) and $4 \pi r^{2} t_{w}(r)$, respectively, $t_{w}(r)$ being the von Weizsäcker density [10].

uncorrelated von Weizsäcker form $4 \pi r^{2} t_{w}(r)$. The example chosen corresponds to $a=2^{-1 / 2}$ a.u. and for the inverse square-law model with $\alpha=1$. In that case, $t_{g}(r)$ can be evaluated analytically as

$$
4 \pi r^{2} t_{g}(r)=4 \pi r^{2} \frac{\left(2-r^{2}+2 r^{4}\right)}{6 \pi^{3 / 2}} e^{-r^{2}}
$$

while $4 \pi r^{2} t_{w}(r)$ has been calculated, for comparison, with the same density $n(r)$ using $t_{w}=(1 / 8) n^{\prime 2} / n$. The effect of interfermion interaction is very appreciable as seen from Fig. 2. This result can be expressed immediately in terms of the probability density $P(r)=\Psi_{R}^{2}(r)$ of the relative motion.

But again, from Eq. (5), $P(r)$ is the Fourier transform of the product of $f(G) \exp \left(\frac{G^{2} a^{2}}{4}\right)$, so that Eq. (B1) is immediately characterized by the density $n(r)$ and, via Eq. (4), its Fourier transform $f(G)$. Equation (B1) evidently therefore provides a concrete example of a major theme of the present article [compare also the formal Euler-Lagrange Eq. (29)]. Explicitly, for $\alpha=2$ in Eq. (B1), the scattering factor $f(G)$ is known to be [5]

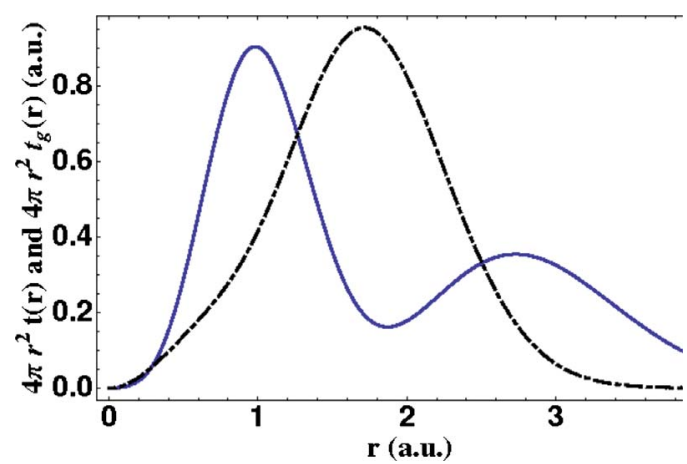

FIG. 3. (Color online) Comparison of different radial kineticenergy densities $4 \pi r^{2} t(r)$ and $4 \pi r^{2} t_{g}(r)$ for the inverse square-law model with $\alpha=1 . t(r)$ (continuous curve) is taken from Eq. (18), while $4 \pi r^{2} t_{g}(r)$ is given in Eq. (B9). The areas under the two curves, of course, are equal, corresponding to the same total kinetic energy. Atomic units are used; the length $a$ being chosen as $2^{-1 / 2}$. 


$$
f(G)=2\left[1-\frac{G^{2} a^{2}}{3}+\frac{G^{4} a^{4}}{60}\right] \exp \left(-\frac{G^{2} a^{2}}{2}\right) .
$$

To conclude this appendix, we have compared in Fig. 3 the two definitions of kinetic-energy density given in Eqs. (18) and (B9). Of course, the total kinetic energy is the same from both equations, that is, $\int t_{g}(r) d \mathbf{r}=\int t(r) d \mathbf{r}$.

\section{APPENDIX C: EFFECT OF INTERPARTICLE INTERACTION ON THE DIFFERENTIAL EQUATION GENERATED BY THE EXTERNAL POTENTIAL ALONE}

The present appendix retains a general confinement potential energy $V(r)$ and also an interparticle interaction $u\left(r_{12}\right)$. Then the arguments of March et al. in [7] are readily employed to yield, with $\hat{\mathbf{r}}$ denoting the unit vector $\mathbf{r} / r$,

$$
\begin{gathered}
\hat{\mathbf{r}}\left[n^{\prime \prime \prime}(r)+\frac{2}{r} n^{\prime \prime}(r)-\frac{2}{r^{2}} n^{\prime}(r)\right]+24[E-V(r)] \int \Psi \nabla_{\mathbf{r}} \Psi d \mathbf{r}_{2} \\
-24 \int\left(\nabla_{\mathbf{r}} \Psi\right) V\left(r_{2}\right) \Psi d \mathbf{r}_{2}+8 E \int \Psi\left(\nabla_{\mathbf{r}} \Psi\right) d \mathbf{r}_{2} \\
\quad-\frac{\partial V(r)}{\partial r} \int \Psi\left(\nabla_{\mathbf{r}} \Psi\right) d \mathbf{r}_{2}+24 \int\left(\nabla_{\mathbf{r}} \Psi\right)\left(\nabla_{\mathbf{r}}^{2} \Psi\right) d \mathbf{r}_{2} \\
\quad+8 \int \Psi \nabla_{\mathbf{r}}\left(\nabla_{\mathbf{r}}^{2} \Psi\right) d \mathbf{r}_{2}-4 \int \Psi V\left(r_{2}\right)\left(\nabla_{\mathbf{r}} \Psi\right) d \mathbf{r}_{2} \\
\quad=32 \int \Psi u\left(\left|\mathbf{r}-\mathbf{r}_{2}\right|\right)\left(\nabla_{\mathbf{r}} \Psi\right) d \mathbf{r}_{2} .
\end{gathered}
$$

This equation separates quite generally the external potential terms on the left from the electron-electron term on the right and is therefore applicable to nonrelativistic He-like ions with nuclear charge $Z e$. However, the ground-state energy $E$ now enters.
[1] N. H. March and W. H. Young, Proc. Phys. Soc. London 72, 182 (1958)

[2] L. Delle Site, J. Phys. A 39, 3047 (2006).

[3] P. A. M. Dirac, Proc. Cambridge Philos. Soc. 26, 376 (1930).

[4] A. Holas, I. A. Howard, and N. H. March, Phys. Lett. A 310, 451 (2003)

[5] A. Akbari, N. H. March, and A. Rubio, Phys. Rev. A 76, 032510 (2007).

[6] N. H. March, A. Akbari, and A. Rubio, Phys. Lett. A 370, 509 (2007).

[7] N. H. March, J. Negro, and L. M. Nieto, J. Phys. A 39, 3741 (2006).

[8] A. Holas and N. H. March, Phys. Rev. A 51, 2040 (1995).
[9] M. Moshinsky, Am. J. Phys. 36, 52 (1968).

[10] C. F. von Weizsäcker, Z. Phys. 2, 376 (1935).

[11] C. Schwartz, Ann. Phys. (N.Y.) 6, 156 (1959).

[12] T. Gál, N. H. March, and Á. Nagy, Chem. Phys. Lett. 305, 429 (1999).

[13] N. H. March, Phys. Lett. A 306, 63 (2002).

[14] R. Crandall, R. Whitnell, and R. Bettega, Am. J. Phys. 52, 438 (1984).

[15] P. Capuzzi, N. H. March, and M. P. Tosi, J. Phys. A 38, L439 (2005).

[16] G. B. Arfken and H. J. Weber, Mathematical Methods for Physics, 5th ed. (Academic, New York, 2001), p. 855. 